\title{
Investigation of the influence of perforation parameters on the acoustic efficiency of sound- absorbing structures
}

\author{
Aleksandr N. Anoshkin ${ }^{1}$, Pavel V. Pisarev ${ }^{1}$, and Karina A. Maksimova ${ }^{1, *}$ \\ ${ }^{1}$ Perm National Research Polytechnic University, 614000 Komsomolsky Prospekt, 29, Russia
}

\begin{abstract}
Within the framework of this work, calculation and experimental studies of the influence of technological defects on the acoustic efficiency of sound-absorbing structures (SAS) are carried out. In this work, the physical and mathematical models of predicting the effective acoustic properties of cells with different diameter of the throat were formulated. A series of laboratory experiments was performed to evaluate the acoustic efficiency of SAS cells with different diameters, on an interferometer with a normal sound wave drop. Based on the results of the studies, the dependences of the natural frequency of the resonator and the coefficient of acoustic pressure loss depending on the diameter of the perforation were revealed.
\end{abstract}

\section{Introduction}

At present, the ecology of aviation transport has become the second most urgent problem, allocated by the International Civil Aviation Organization (ICAO), giving way to the first place only in flight safety. One of the dominant components of the harmful impact of aviation on the environment is the noise on the terrain created by aircraft. One of the most effective ways to reduce the noise of aircraft propulsion systems is to include soundabsorbing structures (SAS) $[1,2]$.

In the process of manufacturing SAS, it is possible to form various technological irregularities or defects. The most common defects are a decrease or an increase in the diameter of the perforation holes. The formation of defects leads to an increase in the spread of acoustic parameters during testing. This leads to more complicated processing of the obtained test data, as well as to the unreliability of the results obtained.

Within the framework of the present work, calculation and experimental studies of the influence of technological defects in the form of deviations of the perforation diameter on the acoustic efficiency of the SAS were carried out.

* Corresponding author: karina-maksimova0402@yandex.ru 


\section{Experimental determination of the acoustic characteristics of the samples of SAS with perforation defects}

For the experiments on the interferometer, model samples of SAS with a diameter of 30 $\mathrm{mm}$ were made, using 3D printing with different hole diameters of $1.4 \div 2.8 \mathrm{~mm}$ (Fig. 1, b). The base diameter was $2.2 \mathrm{~mm}$. To model the defect, as the deviation of the diameter, the following conditions were set: a reduction of $0.8 \mathrm{~mm}$ in $0.2 \mathrm{~mm}$ increments, which is due to the permissible drill wear values and deformation ("shrinkage") of the holes in the carbon plastic; an increase of $0.6 \mathrm{~mm}$ was chosen by the results of technological experiments for manual drilling and is due to the breaking of the holes.

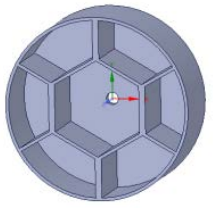

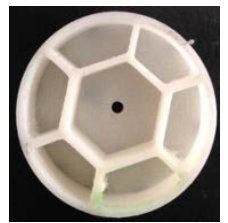

b

Fig. 1. Basic geometric model: a - model sample, b - printed sample.

The production of samples using the $3 \mathrm{D}$ printing method was carried out using FDM prototyping technology, and Figure 2 shows the general view of the Dimension SST 1200 machine used for making samples for the interferometer. The production cycle, in this case, is reduced to the design of the sample shape in the 3D modeling program and the direct printing of the finished sample on the printer (Fig. 3).

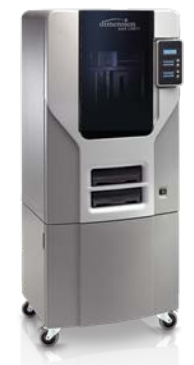

Fig. 2. 3D printer Dimension SST 1200.

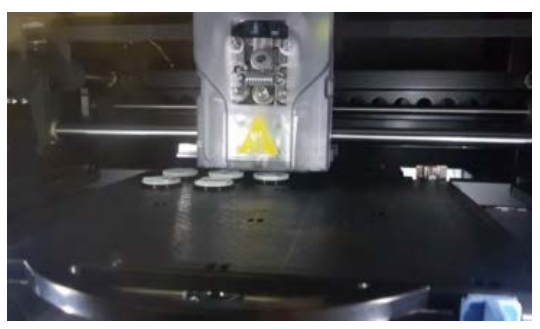

Fig. 3. Making samples on a 3D printer.

Experimental acoustic studies of the samples of the SAS were carried out on the interferometer of the laboratory of noise generation mechanisms and modal analysis of PNRPU (Fig. 4). The experimental equipment is a tube of circular cross-section, at one end of which there is a sample of a SAS, on the other - a speaker that irradiates the sample with acoustic waves. Also at some distance from the sample, there are microphones that record the acoustic pressure of the incident and reflected waves in time. Further, the recorded pressure is processed according to a special procedure, as a result of which the impedance of the SAS sample is calculated. Usually two microphones are used for measurements, since the "transfer function method" used to determine the impedance is the simplest for calculations [3]. 


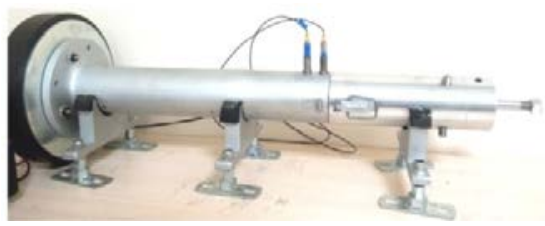

Fig. 4. Fully equiped and ready-to-operate interferometer.

As one of the main problems that arise when determining the acoustic characteristics of a SAS, is the spread of acoustic characteristics. This spread is due to the non-symmetric structure of the resonators, hence, the interference of the waves inside the resonator will depend on the orientation of the cell. To determine the boundary values, a method for determining the spread of acoustic characteristics for a given type of sample was developed. The method assumes a complex of model experiments to determine the dependence of acoustic characteristics on the position of the sample. To implement model experiments to identify the spread on the sample, special marking was made with a step of 30 degrees. Figure 5 shows the test results for the basic version.
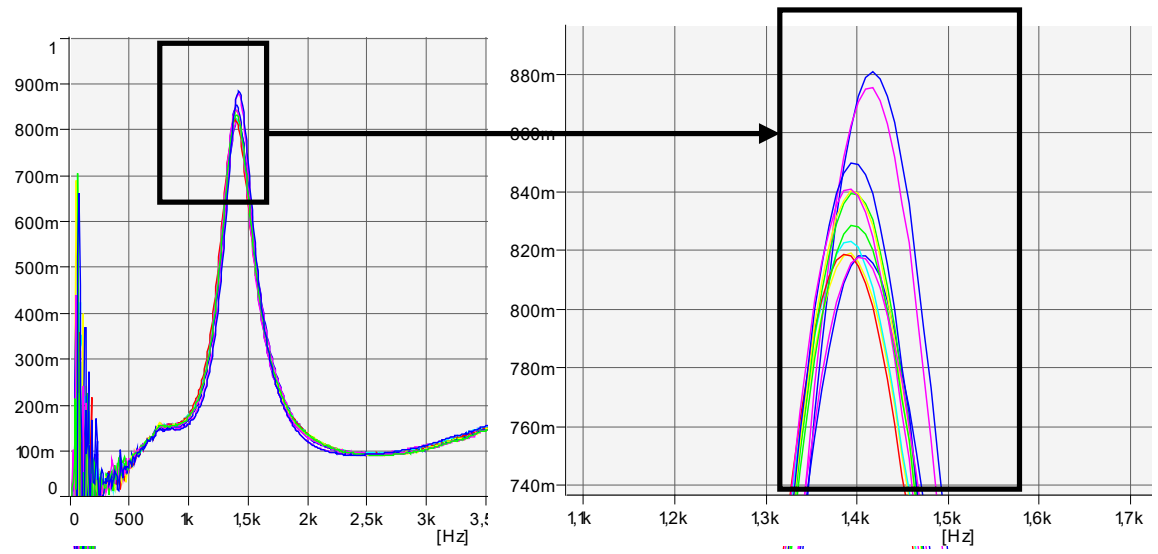

Fig. 5. Dependence of the sound absorption coefficient on the frequency at different positions of the cell.

Analysis of the results revealed that the mean absorption coefficient of the prismatic cavity is 0.85 , and the frequency of $1946 \mathrm{~Hz}$ with a maximum spread of $\alpha=0,04$, resonant frequency $16 \mathrm{~Hz}$. As a working position, the position $180^{\circ}$ was chosen, at which the value of the absorption coefficient corresponds to the average. During subsequent laboratory experiments, the orientation of the sample relative to the measuring part (microphones) was monitored at $180^{\circ}$.

Based on the results of laboratory experiments, the dependence of the sound absorption coefficient on frequency was determined. Figure 6 shows a plot of the acoustic efficiency versus frequency for different hole diameters. In Figure $7 \mathrm{a}, \mathrm{b}$ are graphs of the dependance of own frequency of the resonator from the perforation diameter and dependance of resonator acoustic efficiency from perforation diameter, respectively. 


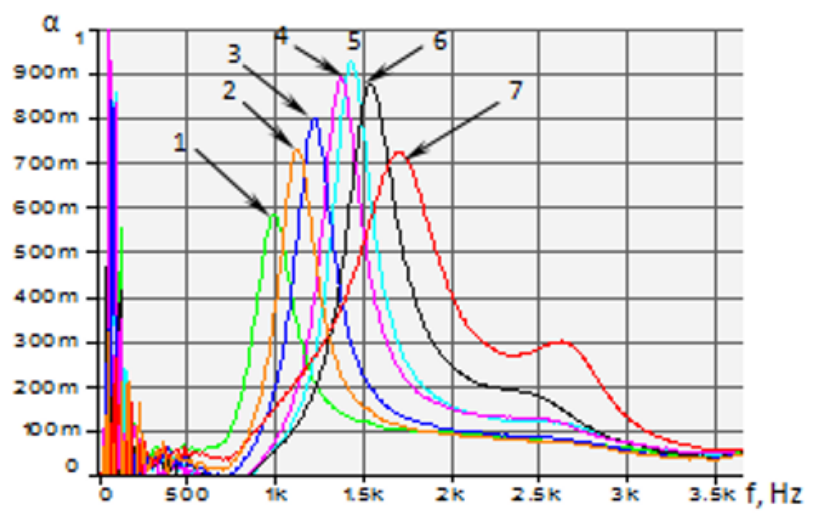

Fig. 6. Dependence of the sound absorption coefficient on frequency: 1 - perforation diameter $=1.4$ $\mathrm{mm} ., 2$ - perforation diameter $=1.6 \mathrm{~mm} ., 3$ - perforation diameter $=1.8 \mathrm{~mm}$., 4 - perforation diameter $=2.0 \mathrm{~mm}, 5$ - perforation diameter $=2.2 \mathrm{~mm}$., 6 - perforation diameter $=2.4 \mathrm{~mm}$., 7 - perforation diameter $=2.6 \mathrm{~mm}$.

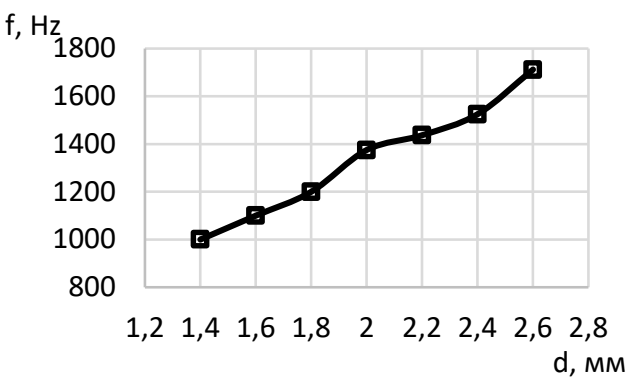

a

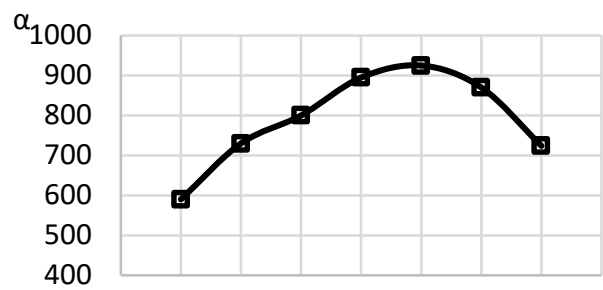

$\begin{array}{lllllllll}1,2 & 1,4 & 1,6 & 1,8 & 2 & 2,2 & 2,4 & 2,6 & 2,8\end{array}$

d, $M M$

b

Fig. 7. Graphs of the dependence: a - the natural frequency of the resonator from the diameter of the perforation; $b$ - the acoustic efficiency of the resonator from the diameter of the perforation.

Dependence analysis revealed that as the diameter of the cavity increases, the resonator's natural frequency increases linearly, with an increase in the diameter of the perforation by $47 \%$ from $1.2 \mathrm{~mm}$ to $2.6 \mathrm{~mm}$, the natural resonator frequency increases by $32 \%$. When the diameter decreases from $2.2 \mathrm{~mm}$ to $1.2 \mathrm{~mm}$, the natural resonator frequency falls by $28 \%$. With an increase in the diameter of the perforation from $2.2 \mathrm{~mm}$ to $2.6 \mathrm{~mm}$, the natural frequency increases by $20 \%$. In addition, an analysis of the results obtained showed that a change in the diameter of the neck affects its broadband.

Analysis of the dependence of the acoustic efficiency of the resonator on the diameter of the perforation revealed that the maximum value of the sound absorption coefficient is observed at a base value of $2.2 \mathrm{~mm}$ in diameter. When the diameter is reduced from $2.2 \mathrm{~mm}$ to $1.2 \mathrm{~mm}$, the sound absorption coefficient decreases by $35 \%$. With an increase in the diameter of the perforation from $2.2 \mathrm{~mm}$ to $2.6 \mathrm{~mm}$, a decrease in the sound absorption coefficient by $24 \%$ is also observed. 


\section{Numerical studies of the acoustic characteristics of the SAS}

In the framework of numerical experiments, the value of the damping effect produced by the prismatic cells, which is a Helmholtz resonator with a different throat diameter, was calculated in the operating frequency range of 1000-4000 Hz.

To perform a number of computational experiments, geometric models were constructed, which consist of a circular channel (free volume of the interferometer) and an attached, free volume of the cell (resonator). Figure 8 shows a general view of the basic geometric model where: 1 - a channel of finite length of circular section, in the center on one of the ends there is a cell - 2 of a prismatic shape, connected with the channel "narrow" throat - 3 cylindrical forms.

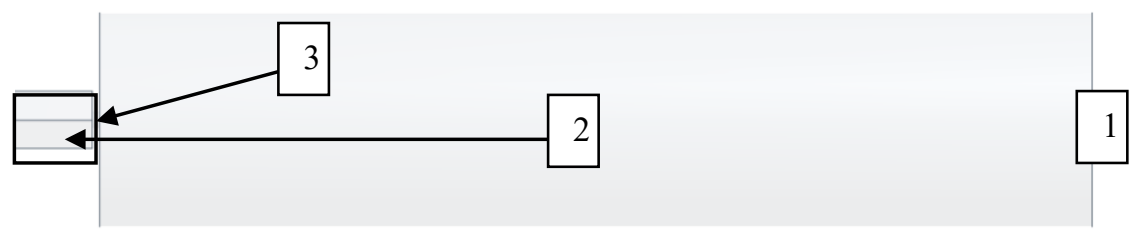

Fig. 8. General view of the geometric model.

The mathematical formulation of the problem of calculating the acoustic characteristics of a resonator involves the Helmholtz equation $[2,4]$ :

$$
-\nabla \cdot\left(\frac{\nabla P}{\rho_{0}}\right)-\frac{\omega^{2}}{\rho_{0} c^{2}} P=0, \text { где } \omega=2 \pi f
$$

where $p$ is the initial pressure in the calculated region ( $1 \mathrm{~atm}$.), $\omega=2 \pi v$ is the natural frequency of the resonator, $\rho_{0}$ is the air density, $c$ is the sound velocity in the environment, and $v$ is the signal frequency at the input.

The wave at the input to the model channel was given as a harmonic pressure wave with amplitude $p_{0}$.

$$
n \cdot\left(\frac{\nabla P}{\rho_{0}}\right)=\frac{i \omega}{\rho_{0} c} P-\frac{2 i \omega}{\rho_{0} c} P_{0}
$$

The output wave was described as:

$$
n \cdot\left(\frac{\nabla P}{\rho_{0}}\right)=\frac{i \omega}{\rho_{0} c} P_{0}
$$

The rigid wall of the model channel was described as:

$$
n \cdot\left(\frac{\nabla P_{0}}{\rho_{0}}\right)=0
$$

The loss of acoustic pressure at the output from the model channel was determined as:

$$
\begin{aligned}
& \mathrm{TL}=10 \log \left(\frac{P_{\text {in }}}{P_{\text {out }}}\right) \\
& P_{\text {in }}=\int_{\partial \Omega} \frac{p_{0}^{2}}{2 p c_{S}} \mathrm{dA}
\end{aligned}
$$




$$
P_{\text {out }}=\int_{\partial \Omega} \frac{\left|p_{c}^{2}\right|}{2 p c_{s}} \mathrm{dA}
$$

Based on the results of the computational experiments, the dependences of the loss of acoustic pressure (TL) on frequency were obtained, and the dependences of the natural frequency of the resonator and the coefficient of loss of acoustic pressure on the diameter of the neck were constructed (Fig. 9).

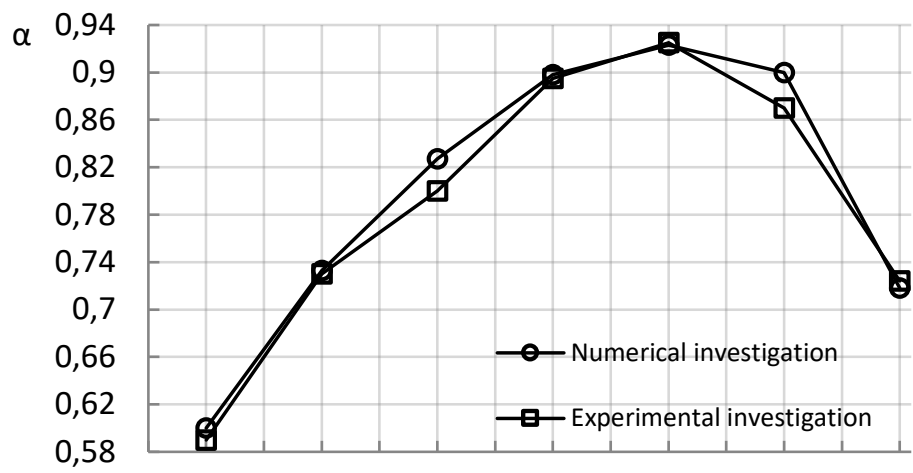

$\begin{array}{lllllllllllllll}1,3 & 1,4 & 1,5 & 1,6 & 1,7 & 1,8 & 1,9 & 2 & 2,1 & 2,2 & 2,3 & 2,4 & 2,5 & 2,6\end{array}$

d, $M M$

Fig. 9. Graphs of the dependence of the acoustic efficiency of the resonator on the diameter of the perforation for numerical and experimental studies.

Analysis of the results of numerical experiments showed that with a decrease in diameter from $2.2 \mathrm{~mm}$ to $1.2 \mathrm{~mm}$, the sound absorption coefficient decreases by $33 \%$. With an increase in the diameter of the perforation from $2.2 \mathrm{~mm}$ to $2.6 \mathrm{~mm}$, a decrease in the sound absorption coefficient by $24.5 \%$ is also observed. Comparative analysis of the results of numerical calculations, with the results of laboratory tests, revealed that the maximum discrepancy is observed for the design version with a diameter of $2.4 \mathrm{~mm}$ and is $3.5 \%$. The obtained error indicates the validity of the developed mathematical numerical model. In the future, the developed numerical model will be used to assess the effect of technological defects on the deviation of the perforation diameter in the SAS with cells of different shapes.

\section{Conclusion}

Thus, it was found that technological defects in the form of a decrease or increase in the diameter of the perforation lead to a significant decrease in the acoustic efficiency of the SAS, namely: 1) Reduction of the diameter of the perforation leads to a significant decrease in the acoustic efficiency of the SAS: the maximum decrease in $\alpha$, taking into account the wear of the drill and shrinkage of the holes reaches 34\%. In addition, the operating frequency is reduced by $400 \mathrm{~Hz}$ (approximately $30 \%$ of the set frequency), which is accompanied by a decrease in the broadband of the PID. 2) With an increase in the diameter of the perforation, the acoustic efficiency of the SAS is also decreased, the maximum decrease in $\alpha$ during the "breaking" of the holes reaches $22 \%$. In addition, there is an increase in the operating frequency by $400 \mathrm{~Hz}$, with a simultaneous increase in the broadband of the SAS. When designing new structures, it is necessary to take into account the effect of defects on the resonance frequency and efficiency of the SAS when it is tuned to the tonal composition of the aircraft engine noise. To prevent technological dispersion of 
hole diameters, it is recommended to use automatic perforation methods, as well as to select the optimal cutting conditions for the laminated PCMs, which prevent deformation of the casing material and tool wear.

The research was carried out at the Perm national research polytechnic university with the support of the state task (Project No. 9.7955.2017 / 9.10).

\section{References}

1. A.G. Zakharov, A.N. Anoshkin, A.A. Pankov, P.V. Pisarev, Bulletin of the PNRPU. Aerospace Engineering, 46, 144-159 (2016)

2. P.V. Pisarev, A.N. Anoshkin, A.A. Pan'kov, AIP Conf. Proc., 1770 (2016)

3. O.Yu. Kustov, V.V. Palchikovsky, Aerospace engineering, high technologies and innovations, 1, 157-160 (2015)

4. A.N. Anoshkin, A.A. Pan'kov, P.V. Pisarev, Scientific and technical volga region bulletin, 1, 354-357 (2015) 